\title{
Interaction of catechol $O$-methyltransferase and serotonin transporter genes modulates effective connectivity in a facial emotion-processing circuitry
}

\author{
SA Surguladze ${ }^{1,2}$, J Radua ${ }^{1,3}$, W El-Hage ${ }^{1,4}$, B Gohier ${ }^{5}$, JR Sato ${ }^{6}$, DM Kronhaus ${ }^{7}$, P Proitsi ${ }^{1}$, J Powell ${ }^{1}$ and ML Phillips ${ }^{8,9}$
}

Imaging genetic studies showed exaggerated blood oxygenation level-dependent response in limbic structures in carriers of low activity alleles of serotonin transporter-linked promoter region (5-HTTLPR) as well as catechol 0-methyltransferase (COMT) genes. This was suggested to underlie the vulnerability to mood disorders. To better understand the mechanisms of vulnerability, it is important to investigate the genetic modulation of frontal-limbic connectivity that underlies emotional regulation and control. In this study, we have examined the interaction of 5-HTTLPR and COMT genetic markers on effective connectivity within neural circuitry for emotional facial expressions. A total of 91 healthy Caucasian adults underwent functional magnetic resonance imaging experiments with a task presenting dynamic emotional facial expressions of fear, sadness, happiness and anger. The effective connectivity within the facial processing circuitry was assessed with Granger causality method. We have demonstrated that in fear processing condition, an interaction between 5-HTTLPR (S) and COMT (met) low activity alleles was associated with reduced reciprocal connectivity within the circuitry including bilateral fusiform/inferior occipital regions, right superior temporal gyrus/superior temporal sulcus, bilateral inferior/middle prefrontal cortex and right amygdala. We suggest that the epistatic effect of reduced effective connectivity may underlie an inefficient emotion regulation that places these individuals at greater risk for depressive disorders.

Translational Psychiatry (2012) 2, e70; doi:10.1038/tp.2011.69; published online 17 January 2012

\section{Introduction}

The last decade has seen the emergence of imaging genetics, as a research strategy to elucidate relationships between genotypic markers and neural structures or processes, that can help to identify pathophysiological processes predisposing to psychiatric disorders. ${ }^{1}$ One consistent finding is exaggerated amygdala response to threat-related stimuli in carriers of low activity short $(S)$ allele of the serotonin transporter-linked promoter region (5-HTTLPR) gene (see review $^{2}$ ). This genetic modulation of brain response to emotionally negative signals has been highlighted as a potential mechanism underlying inefficiency of emotional processing and susceptibility to depressive disorders. ${ }^{3}$ Another key genetic variation modulating emotional processes is the val158met single nucleotide polymorphism (SNP) of the gene coding for the catechol O-methyltransferase (COMT) enzyme that inactivates extraneuronal dopamine. Imaging genetic studies have reported that met carriers overactivated subcortical limbic regions in response to negative emotional stimuli. ${ }^{4-6}$ These results suggest a role for the met allele in predisposing to greater stress reactivity ${ }^{7}$ and a negative emotion attentional bias that may confer risk for affective disorders. Direct evidence for an association between COMT genotype and major depressive disorder (MDD) remains equivocal. ${ }^{8}$ The investigators, however, emphasized the importance of gene-gene interaction (epistasis) in predisposing toward complex syndromes, such as MDD, that may occur even in the absence of main effects of single genes. 9,10 This is supported by the evidence of interactive effects of COMT and 5-HTTLPR genotypes in predisposing toward development of MDD in individuals with a history of stressful life events. ${ }^{11}$

There is little research on interactive effects of the above genetic markers on neural response. To the best of our knowledge, the only imaging genetic study to examine the joint effect of 5-HTTLPR and COMT genotypic variation upon neural activity in healthy individuals demonstrated additive effects of low activity 5-HTTLPR and COMT alleles ( $S$ and met alleles, respectively), resulting in exaggerated limbic activity during the processing of emotionally unpleasant pictures. ${ }^{12}$ This additive effect was suggested to underlie low resilience to dysphoric mood states in individuals carrying low activity alleles of both genes.

\footnotetext{
${ }^{1}$ Institute of Psychiatry, King's College London, London, UK; ${ }^{2}$ Cygnet Health Care, London, UK; ${ }^{3}$ FIDMAG, CIBERSAM, Sant Boi de Llobregat, Barcelona, Spain; ${ }^{4}$ Inserm U930 ERL CNRS 3106, Université François Rabelais, CHRU Tours, France; ${ }^{5}$ UPRES EA2646, Department of Psychiatry, University Hospital of Angers, Angers, France; ${ }^{6}$ Centre of Mathematics, Computation and Cognition - Universidade Federal do ABC, São Paulo, Brazil; ${ }^{7}$ St Catharine's College and Computer Laboratory, University of Cambridge, Cambridge, UK; ${ }^{8}$ Department of Psychiatry, Western Psychiatric Institute and Clinic, University of Pittsburgh School of Medicine, Pittsburgh, PA, USA and ${ }^{9}$ Department of Psychological Medicine, Cardiff University School of Medicine, Cardiff, UK Correspondence: Dr SA Surguladze, Institute of Psychiatry, King's College London, Box PO69, DeCrespigny Park, London SE5 8AF, UK. E-mail: simon.2.surguladze@ $@$ kl.ac.uk Keywords: COMT; effective connectivity; facial emotion processing; 5-HTTLPR Received 15 July 2011; revised 7 December 2011; accepted 11 December 2011
} 
As evidenced by recent studies, the measures of interregional brain connectivity may be more sensitive than the estimates of regional neural activity based on blood oxygenation level-dependent (BOLD) response. ${ }^{13}$ The connectivity between amygdala and anterior prefrontal regions during the processing of negative emotional stimuli is proposed to underlie emotional regulation and control. ${ }^{14,15}$ Importantly, the studies in depression found reduced connectivity between the limbic structures and anterior prefrontal regions, which was suggested to reflect inefficient emotion regulation. ${ }^{16-19}$ This evidence is supporting a neural model of $\mathrm{MDD}^{20}$ that emphasized a functional 'uncoupling' between anterior limbic and prefrontal cortical regions that are critically engaged during emotion regulation.

Findings regarding the impact of COMT and 5-HTTLPR genotypic variations on functional connectivity in healthy individuals are, however, inconsistent. For example, the 5-HTTLPR $S$ allele has been associated with greater functional connectivity between the amygdala and rostral ventromedial prefrontal cortex ${ }^{21}$ but also decreased functional connectivity between the amygdala and subgenual anterior cingulate cortex, ${ }^{14}$ in response to emotionally negative visual stimuli. Similarly, COMT met allele carriers have shown greater functional connectivity between the right amygdala and ventrolateral prefrontal cortex ${ }^{5}$ and right orbitofrontal cortex, ${ }^{22}$ but also reduced effective connectivity between dorsolateral prefrontal cortex and ventral striatum. $^{23}$

The aim of the present study was to examine the joint effect of COMT and 5-HTTLPR functional polymorphisms upon the effective connectivity in neural circuitry, supporting facial emotion processing in healthy volunteers. We employed three novel approaches:

(1) We examined the interaction, as well as separate effects, of COMT and 5-HTTLPR genetic variations upon emotion-processing neural circuitry.

(2) We focused on measures of effective connectivity employing Granger connectivity analysis. ${ }^{24,25}$ This approach can identify the direction of informational flow between neural regions of interest unlike, for example, psychophysiological interaction, that focuses mainly on temporal relationships between the BOLD signal of regions of interest. ${ }^{26}$ As a data-driven approach, Granger effective connectivity analysis also differs from structural equation modeling ${ }^{27}$ and dynamic causal modeling, ${ }^{28}$ that rely on a-priori network model specifications. $^{29,30}$

(3) We employed a novel ecologically relevant paradigm that comprised dynamic facial emotional expressions. Previous studies have reported that neural regions supporting face emotion processing, including the amygdala and fusiform gyrus, were more strongly activated by dynamic vs static emotional faces. ${ }^{31}$ Based on previous findings, we hypothesized that the carriers of low activity COMT and 5-HTTLPR genotypes will demonstrate a pattern of inefficient emotion regulation represented by reduced Granger effective connectivity from prefrontal cortical to anterior limbic regions in response to threat-related facial emotions.

\section{Methods}

Participants. A total of 91 right-handed white Caucasian healthy individuals (45 female; age $=32.5 \pm 9$, range 19-56 years) with no personal or family history of psychiatric disorder participated in four fMRI experiments. Exclusion criteria were current or past psychiatric diagnosis as established by the Structured Clinical Interview for DSM IV (SCID) screen. ${ }^{32}$

The study was approved by the Ethics Committee of the Institute of Psychiatry, King's College London, UK. Participants were provided with full details about the experimental protocol and gave their written informed consent before the beginning of the experiment.

Genotyping. The polymorphism val158met was considered for COMT genotyping, in line with the suggestions ${ }^{33}$ based on direct comparisons of the effects of single COMT val158met SNP (rs4680) vs the haplotypes employed in previous studies. ${ }^{34,35}$ It was demonstrated that the val158met polymorphism was more informative for understanding the effect of COMT on neural activity when compared with the haplotypes used by the above investigators. ${ }^{34,35} \mathrm{We}$ emphasize, however, that the findings of the comparison study $^{33}$ do not imply that haplotype-based models are generally inferior to models based on individual SNPs. In our case, the use of COMT val158met SNP was preferable in order to avoid unnecessary model complexity when applying an interaction analysis to the multiple connectivity data.

Regarding the 5-HTTLPR gene, we considered both the well established difference between the higher expression $5-H T T L P R$ long $(L)$ allele vs the low expression $S$ allele, ${ }^{36}$ and the evidence regarding the impact of the rs25531 G/A SNP upon functioning of the $L$ allele. ${ }^{37}$ We reclassified 5-HTTLPR alleles on the basis of lower and higher levels of expression similarly to previous reports. ${ }^{38}$ Thus, $L G$ and $S$ were recoded as $S^{\prime}$, whereas $L A$ and $L$ were recoded as $L$ '.

DNA was extracted from cheek swabs using standard procedures (see Supplementary Method in the online supplement).

The COMT met/met homozygosity was detected in 29 participants and val/val in 20 participants; 41 participants were heterozygous. After recoding the 5-HTTLPR alleles, as indicated above, 26 participants were homozygous $L ' / L$ ', 22 participants were homozygous $S$ '/S' and 36 participants were heterozygous. There was no statistical deviation from HardyWeinberg equilibrium for all polymorphisms. The proportions of COMT and 5-HTTLPR low activity genotypes were independent from each other (Spearman correlation between the number of COMT met alleles and the number of 5-HTTLPR S' alleles: $\rho=-0.049, P=0.647$ ). Due to low DNA yield, the data on COMT did not pass the quality control in one participant, and, on 5-HTTLPR, in seven participants.

As the missing genotyping values represented only a small proportion $(<5 \%)$ of the whole data, to analyse the genetic effect on neural response and connectivity, we have employed the imputation approach, that is, genotyping data were filled in with the expectation maximization algorithm of the 'gc.em' procedure for R. ${ }^{39}$ 
Neuroimaging paradigm. There were four experimental runs, one per each of four facial expressions: fear, anger, sadness or happiness. The active condition stimuli were short monochromatic movie excerpts generated from the NimStim series of facial pictures (http://www.macbrain.org/), as described elsewhere. ${ }^{40}$ In particular, in each excerpt, the facial expression changed from neutral to the emotional one over a period of $1 \mathrm{~s}$. The models were males and females of Caucasian, African or Asian origin. Gender and racial distribution were similar in all emotional experiments. In each of the four emotional experimental runs, there were nine blocks of 12 movie clips per block. Each block lasted $42 \mathrm{~s}$. Three of these blocks displayed facial expressions. The other blocks comprised either baseline (three blocks) or 'identity morph' (three blocks) conditions. The baseline blocks comprised presentation of $1 \mathrm{~s}$ movie excerpts containing monochromatic ovals. The ovals were approximately of the same size as that of the models' faces in the facial expression blocks. To control for the dynamic aspect of the facial expressions in active condition, each oval contained a smaller dynamic one with darker borders, concentrically expanding/moving from center to the perimeter. The third condition ('identity morphs') block contained $1 \mathrm{~s}$ movie excerpts in which one model with a nonemotional, neutral expression changed dynamically to another identity displaying a neutral expression. We did not include the analysis of identity morph blocks in the present study, as our focus was to compare activity and effective connectivity in facial expression relative to baseline blocks. To ensure that the participants were attending to the stimuli (in the second half of each movie excerpt a colored translucent filter (either orange, blue or yellow) appeared for $300 \mathrm{~ms}$ ), the participants were requested to press the button with their right index finger as soon as they saw the color filter. To avoid habituation, the interstimulus interval between the movie clips was 'jittered', varying from 2000 to $2999 \mathrm{~ms}$, mean $=2500 \mathrm{~ms}$. Each experimental run (in which one of the four facial expression types was presented) lasted $6 \mathrm{~min}$ and $18 \mathrm{~s}$. The participants completed all four experiments within the same session, with short breaks between them, during which they stayed in scanner, listening to the researcher reminding them the instructions through the intercom. The order of the emotional conditions was counterbalanced between participants.

Neuroimaging data acquisition. Scanning was performed on a GE Signa 3 Tesla scanner (Milwaukee, WI, USA). Reliable image quality was obtained by using a semi-automated quality control procedure. For BOLD imaging, 189 T2*-weighted whole-brain volumes were acquired during the experimental conditions. The EPI data set was acquired parallel to the intercommissural plane and consisted of 38 slices: $\mathrm{TR}=2000 \mathrm{~ms}, \mathrm{TE}=25 \mathrm{~ms}$, flip angle $=80^{\circ}$, slice thickness $=$ $2.4 \mathrm{~mm}$, inter-slice gap $=1.0 \mathrm{~mm}$, image acquisition matrix size $=64^{2}$. High-resolution structural images comprised 43 slices with slice thickness/gap $3.0 / 0.3 \mathrm{~mm}, T R=3000 \mathrm{~ms}$, $\mathrm{TE}=30 \mathrm{~ms}$, flip angle $=90$ and matrix size $=128^{2}$.

\section{fMRI data analysis}

a. The BOLD response to each emotional condition was analyzed with XBAM v4 (Institute of Psychiatry, London,
UK), which is based on permutation testing that allows a mixed effects approach to analysis, ${ }^{41}$ see also http:// brainmap.it.

b. To identify the neural circuit responding to any of the four emotional conditions (general emotion-processing circuit), a binary map was produced where the BOLD responses to all four emotional expressions overlapped. This was necessary-in order to investigate the genetic effects on the same neural circuit across different emotional conditions.

c. Effective connectivity was examined based on Granger causality analysis that provides estimates of the direction of information flow between the nodes of the neural circuit. This information flow is estimated by the analysis of temporal precedence and could be either uni- or bidirectional (reciprocal). We have used cluster Granger analysis, ${ }^{30}$ which is an extension of the original concept. $^{24,25}$ To deal with the high dimensionality of fMRI data, the cluster Granger analysis is employing principal component analysis, thus accounting for multiple time-series within each region of interest, rather than being related to the peak voxel or to the average time-series of the voxels within the region of interest. Cluster Granger analysis is using canonical correlations, which is also different from the recently introduced multivariate Granger analysis. ${ }^{42}$

d. To compare the strength in effective connectivity between individuals with different genotypes, we applied the measure of the 'total-degree'. This measure, in analogy to graph theory, ${ }^{43}$ was defined as the total number of significant Granger-causalities in the general emotionprocessing circuit. For instance, if Granger analyses in a given individual had detected 36 statistically significant Granger-causalities between the regions of interest, their total-degree was 36 . When comparing genotypes, we looked at the statistical difference in total-degrees between the groups of individuals with different genetic polymorphisms.

\section{Results}

General emotion-processing circuit. Activation maps pertaining to processing of each of four emotional conditions showed the regions that were either emotiongeneral or relatively specific to each emotion (Table 1). The emotion-general map, where the clusters overlapped across all four conditions, included six neural regions: bilateral fusiform/inferior occipital regions (FOG, BA 19, 37, 18), right superior temporal gyrus/superior temporal sulcus (RSTG, BA 21, 22 and 39), bilateral inferior/middle prefrontal cortex (IFG, BA 9, 44, 45) and right amygdala (RAMG), Figure 1. The regions that were differentially activated in each condition, in addition to the general emotion-processing circuit, are shown in Supplementary Table S1.

Effects of genotypic variation in 5-HTTLPR and COMT genes upon BOLD response in the general emotionprocessing circuit. There were no significant effects of either 5-HTTLPR or COMT genetic polymorphisms on BOLD signal in any of the six regions within the general emotion- 
Table 1 Activation regions detected in different emotional conditions and the overlap regions

\begin{tabular}{|c|c|c|c|}
\hline & \multirow{2}{*}{$\begin{array}{l}\text { Number } \\
\text { of voxels }\end{array}$} & \multicolumn{2}{|c|}{ Maxima } \\
\hline & & $\begin{array}{c}\text { Talairach } \\
x, y, z^{a}\end{array}$ & $\underset{P}{\text { Corrected }}$ \\
\hline \multicolumn{4}{|l|}{ Fearful faces vs ovals ${ }^{\mathrm{b}}$} \\
\hline Bilateral temporo-occipito-cerebellar region BA 19, 37 & 545 & $\begin{array}{r}32,-70,-16 \\
-36,-70,-23\end{array}$ & $\begin{array}{l}0.0002 \\
0.0002\end{array}$ \\
\hline Right MFG region, BA 6, 10 & 431 & $7,63,10$ & 0.0002 \\
\hline Left IFG/middle frontal region, BA 6, 46 & 160 & $-43,33,13$ & 0.0007 \\
\hline Right ventral striatum, amygdala & 33 & $22,4,-13$ & 0.001 \\
\hline \multicolumn{4}{|l|}{ Angry faces vs ovals ${ }^{\mathrm{b}}$} \\
\hline Bilateral temporo-occipito-cerebellar region BA 36 & 1000 & $\begin{array}{r}36,-41,-20 \\
-32,-67,-20\end{array}$ & $\begin{array}{l}0.0002 \\
0.0002\end{array}$ \\
\hline Bilateral IFG/middle prefrontal region, BA 6, 9 & 388 & $\begin{array}{r}40,15,26 \\
-43,0,30\end{array}$ & $\begin{array}{l}0.0002 \\
0.0005\end{array}$ \\
\hline Right ventral striatum, amygdala & 48 & $18,4,-13$ & 0.0005 \\
\hline \multicolumn{4}{|l|}{ Happy faces vs ovals ${ }^{\mathrm{b}}$} \\
\hline Bilateral temporo-occipito-cerebellar region, BA 19, 37 & 778 & $\begin{array}{r}40,-67,-13 \\
-40,-70,-20\end{array}$ & $\begin{array}{l}0.0003 \\
0.0003\end{array}$ \\
\hline Bilateral middle frontal/precentral region, BA 9,46 & 624 & $\begin{array}{r}43,30,13 \\
-40,4,33\end{array}$ & $\begin{array}{l}0.0003 \\
0.0003\end{array}$ \\
\hline Left STG/STS region, BA 22 & 51 & $-51,-44,3$ & 0.001 \\
\hline Right parahippocampal region, amygdala & 26 & $25,0,-13$ & 0.001 \\
\hline \multicolumn{4}{|l|}{ Sad faces vs ovals ${ }^{\mathrm{b}}$} \\
\hline Bilateral IFG/precentral region, BA 6,45 & 1114 & $\begin{array}{r}32,7,26 \\
-36,26,7\end{array}$ & $\begin{array}{l}0.0002 \\
0.0002\end{array}$ \\
\hline Bilateral temporo-occipito-cerebellar region & 851 & $\begin{array}{r}36,-41,-20 \\
-36,-41,-23\end{array}$ & $\begin{array}{l}0.0002 \\
0.0002\end{array}$ \\
\hline Right parahippocampal region, amygdala & 47 & $22,0,-10$ & 0.0007 \\
\hline Right STG/insula region, BA 13 & 37 & $36,-44,26$ & 0.002 \\
\hline \multicolumn{4}{|l|}{ Overlap regions: general emotion-processing circuit } \\
\hline Bilateral fusiform/occipital regions, BA 18, 19, 37 & 169 & $\begin{array}{r}26,-60,-17 \\
-37,-64,-20\end{array}$ & $\mathrm{~N} / \mathrm{A}$ \\
\hline Right STG/STS, BA 21,22 & 44 & $45,-41,7$ & $\mathrm{~N} / \mathrm{A}$ \\
\hline Bilateral inferior/middle PFC, BA 9,44, 45 & 34 & $\begin{array}{r}41,19,27 \\
-37,7,30\end{array}$ & $\mathrm{~N} / \mathrm{A}$ \\
\hline Right parahippocampal region/amygdala & 5 & $19,-4,-10$ & $\mathrm{~N} / \mathrm{A}$ \\
\hline
\end{tabular}

${ }^{a}$ Coordinates represent the voxel with maximum cluster activation in contrasts between emotional faces and ovals, and the voxel with minimum square distances to the other voxels of the cluster in overlap map.

${ }^{\mathrm{b}}$ Contrasts between emotional faces and ovals were thresholded with voxel $P=0.05$ and cluster $P=0.01$.

processing circuit in any emotional condition. In the condition with fearful expressions only, the BOLD signal in RAMG was higher in low activity polymorphism carriers, although the difference did not reach significance: COMT met/met $>$ val/ val, $P=0.13$ and 5-HTTLPR S'S' $>$ L' $L^{\prime}, P=0.26$.

\section{Effective connectivity in the general emotion-processing} circuit. In each emotional condition, all Granger connectivity pathways in the general emotion-processing circuit were statistically significant in both directions between neural regions (Supplementary Table S2), with no significant directionality difference except for one connection: in the fear condition the connectivity from the RSTG to right FOG was stronger than other way round (McNemar $\chi^{2}=10.3$, $\mathrm{df}=1$, FDR-corrected $P$-value $=0.038)$. However, in this link the effective connectivity in both directions remained highly significant. The effective connectivity in the entire general emotion-processing circuit was significant in each emotional condition (all $P \leqslant 3 \mathrm{E}-09$ ), Figure 2.
Genetic effects upon effective connectivity in the general emotion-processing circuit. There was a significant negative relationship between the number of $S^{\prime}$ alleles of 5-HTTLPR and total effective connectivity in the fearful condition only $(\rho=-0.26, P=0.012)$, but not in the conditions with sad, angry or happy faces. This $S$ '-related progressive reduction of effective connectivity was seen in the contrasts between $L^{\prime} / L^{\prime}$ and $L^{\prime} / S^{\prime}$ genotypes (difference $=5.5 ; W=792, P=0.004$ ), and between $L^{\prime} / L^{\prime}$ and $S^{\prime} / S^{\prime}$ genotypes (difference $=5.9 ; \mathrm{W}=399, P=0.020$ ).

Similarly, for the COMT gene, there was a significant negative correlation between the number of met alleles and total effective connectivity in the fearful condition only ( $\rho=-0.27, P=0.009)$. There were significant differences between $\mathrm{val} / \mathrm{val}$ vs $\mathrm{val} / \mathrm{met}$ carriers (difference $=4.8$; $\mathrm{W}=567, \quad P=0.027)$ and $\mathrm{val} / \mathrm{val}$ vs met $/ \mathrm{met}$ carriers (difference $=6.2 ; \mathrm{W}=441, P=0.002$ ).

We next conducted an ANOVA with the following binary regressors: COMT (val/val vs either val/met or $\mathrm{met} / \mathrm{met}$ ), 

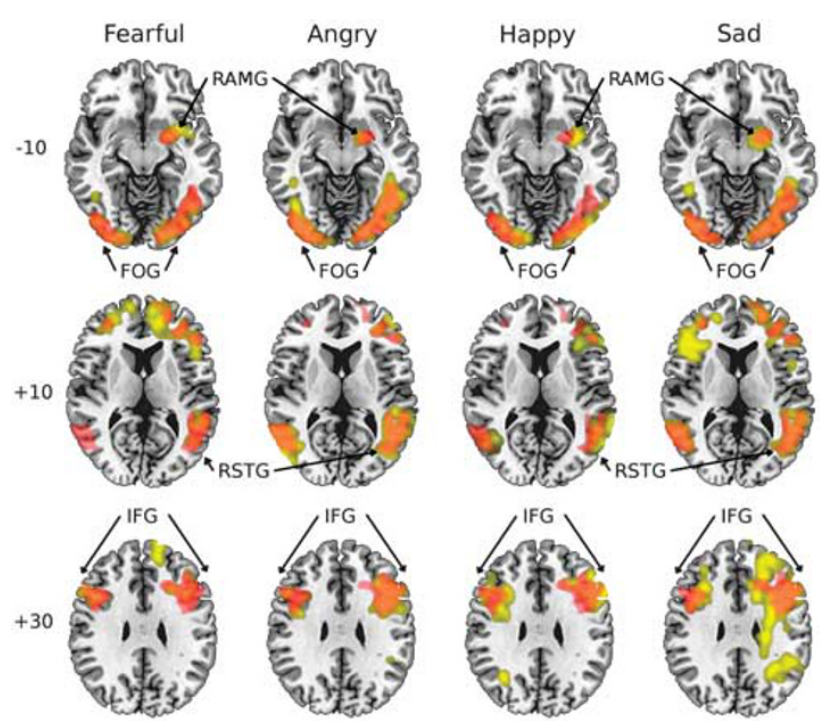

Figure 1 Activation regions detected in different emotional conditions. Axial slices at $z=-10$ (top), +10 (middle) and +30 (bottom) Showing brain regions with significant BOLD response to the facial emotional stimuli. The regions commonly activated by each emotional expression (general emotion-processing circuit) are shown in red. These include: bilateral fusiform/inferior occipital regions (FOG. BA $19 ; 37 ; 18$ ), right superior temporal gyrus/superior temporal sulcus (RSTG. BA 21; 22 and 39) bilateral inferior/middle prefrontal cortex (IFG. BA 9; 44; 45) and right amygdala (RAMG). The regions that were additionally activated by particular emotional expressions are shown in yellow. Left side of the slice corresponds to the left side of the brain.

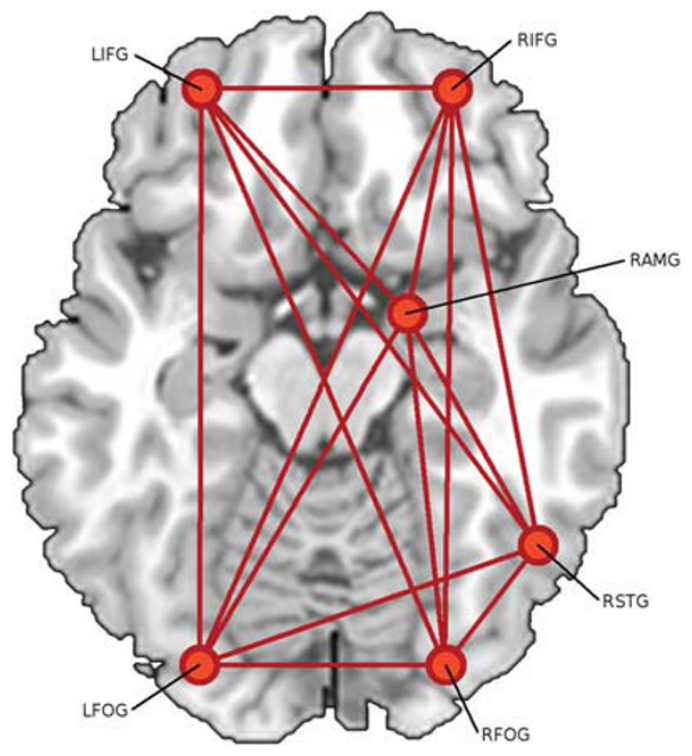

Figure 2 General emotion-processing circuit: fearful faces condition. Schematic depiction of inter-regional connections within the general emotion-processing circuit. Left side of the slice corresponds to the left side of the brain. LIFG, left inferior frontal gyrus; RIFG, right inferior frontal gyrus; RAMG, right amygdala; RSTG, right superior temporal gyrus; LFOG, left fusiform/occipital gyrus; RFOG, right fusiform/ occipital gyrus.

5-HTTLPR ( $L^{\prime} / L^{\prime}$ vs either $L^{\prime} / S^{\prime}$ or $\left.S^{\prime} / S^{\prime}\right)$ and COMT $\mathrm{x}$ 5 -HTTLPR, with total degree connectivity in fear condition as a dependent variable. The interaction model was

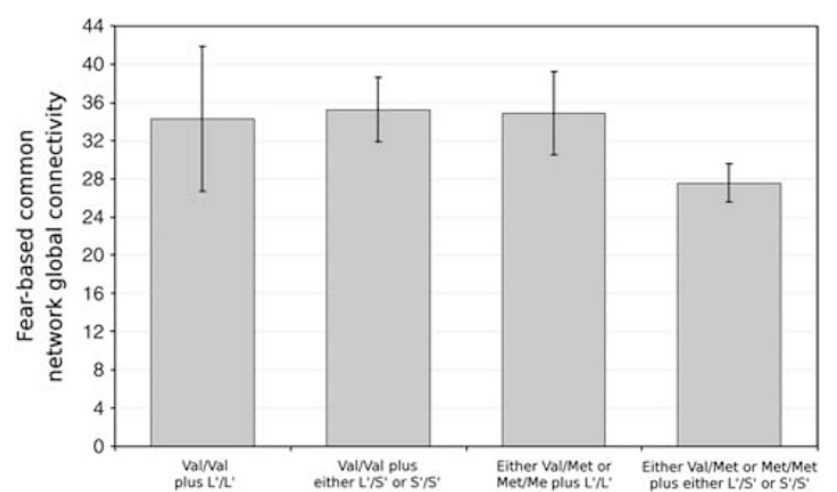

1 (I)

III

IV

Figure 3 Effective connectivity in the general emotion-processing circuit: fearful faces condition. Bars represent the mean total-degree and error bars their $95 \%$ confidence intervals based on Student's $t$-distribution. The effective connectivity in individuals lacking at least one of the homozygotes L'L' or Val/Val (bar IV) is reduced, compared with those carrying any other combinations of alleles (bars I-III).

statistically significant $(F=7.1, \mathrm{df}=3,87, P=0.0003$, adjusted $R^{2}=17 \%$ ). The interaction effect was stronger than an additive one $(\mathrm{F}=4.2, \mathrm{df}=1, P=0.045)$.

The effective connectivity in general emotion-processing circuit was lower only in val/met or $\mathrm{met} / \mathrm{met}$ carriers who at the same time were either $L ' / S^{\prime}$ or $S^{\prime} / S^{\prime}$ carriers (mean totaldegree $=27.6$ ), compared with participants who were homozygous for either val or $L$ 'or were homozygous for both val and $L^{\prime}$ (mean total-degree $=34.9$; difference $=7.3 ; \mathrm{W}=1575$, $P=7$ E-06 (Figure 3 ).

\section{Discussion}

Our findings demonstrated significantly reduced reciprocal effective connectivity in the facial emotion-processing circuit in the carriers of low activity 5-HTTLPR S' and COMT met alleles, relative to those with high activity homozygotes 5HTTLPR L'L' and COMT val/val. Furthermore, there was a significant interaction between 5-HTTLPR and COMT polymorphisms upon effective connectivity in the face emotionprocessing circuit. Effective connectivity was lower in val/met or met/met carriers who at the same time were either $L^{\prime} / S^{\prime}$ or $S ' S^{\prime}$ carriers, compared with individuals who were homozygous for either val or L' alleles, or had both val and L' homozygotes. The above effects were observed in a fear condition, in the circuit comprising bilateral fusiform/occipital regions, bilateral inferior prefrontal cortex, right superior temporal gyrus/superior temporal sulcus and the right amygdala.

The combined effect of 5-HTTLPR and COMT polymorphisms has been studied previously, albeit only with regard to the BOLD response to emotional pictures. ${ }^{12}$ Our results concur, with the proposal of the above study, ${ }^{12}$ that the joint effect of low activity 5-HTTLPR and COMT polymorphisms confers inefficient emotion processing. The novelty of our findings is that the interactive effect of 5-HTTLPR and COMT polymorphisms upon effective connectivity provides an insight into the genetically determined differences in emotion regulation, rather than emotion processing per se. We emphasize that above polymorphisms modulated 
connectivity in a distributed neural circuit, rather than connectivity between a-priori selected prefrontal regions and amygdala. Importantly, although this circuit has been determined empirically, it is fully consistent with existing neural models for face processing. ${ }^{44,45}$

Our results are in line with the findings of decreased connectivity (uncoupling) between anterior prefrontal cortical regions and amygdala in healthy carriers of low activity alleles of $5-H T T L P R$ in response to emotionally aversive stimuli. ${ }^{14}$ Some previous studies have reported increased corticolimbic connectivity in 5-HTTLPR $S$ allele carriers ${ }^{21,46}$ and COMT met/met homozygotes. ${ }^{5,22}$ We believe that our results do not contradict earlier results, given that it has previously been shown that connectivity between the brain regions depends on the precise prefrontal cortical regions examined. For example, one study ${ }^{14}$ demonstrated both increased connectivity between ventro-medial prefrontal cortex and amygdala and decreased connectivity between subgenual ACC and amygdala in the $S$ allele carriers vs $L$ allele homozygotes of 5-HTTLPR. We note here that the prefrontal cortical regions of the emotional circuit identified in our study were located dorsally and laterally to the ventro-medial

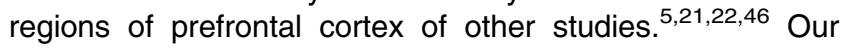
finding that low activity alleles of both 5-HTTLPR and COMT genes (that is, alleles that are thought to confer risk for emotional disorders) are associated with reduced connectivity within a distributed face emotion-processing network are consistent with findings of reduced connectivity between prefrontal and ventral limbic regions in individuals with MDD, ${ }^{16-19,29}$ or pathological anxiety, ${ }^{47}$ including $\mathrm{PTSD}^{48}$ and social anxiety disorder. ${ }^{49}$

What are the implications of the reduced connectivity within the emotion-processing circuit? The converging evidence from animal research, ${ }^{50,51}$ neuroimaging data on healthy individuals $^{14,15,52}$ and the above studies in patients with depression and anxiety disorders indicate that reduced prefrontal-limbic connectivity may underlie inefficient emotion regulation during the processing of negative stimuli. We suggest that our findings of reduced connectivity within the emotion-processing neural circuit may be a necessary, although not sufficient component pathophysiological process in MDD. Indeed, the MDD is known to be associated with cellular and structural abnormalities in prefrontal ${ }^{53}$ and temporal $^{54}$ cortices and/or abnormal reductions in prefrontal cortical activity. ${ }^{55}$

We briefly consider here the issue of directionality as examined with the Granger connectivity analysis. Neuroimaging genetic studies that have explored functional connectivity, found genetic modulation of connectivity between medial prefrontal and limbic regions. ${ }^{14,21}$ This was suggested to indicate a modulation of top-down effect on emotion regulation. However, the assumption has not been formally tested, as functional connectivity is of correlational rather than a causal character. Using Granger effective connectivity, we specifically tested the potentially top-down relationships within the emotion-processing circuit.

Our data demonstrated that the frontal regions did not simply impact on limbic areas in a top-down manner, but there was a rather more complex functional organization that involved reciprocal feed-forward and feedback relationships.
This included reduced bi-directional connectivity between the prefrontal cortex and amygdala in individuals with low activity COMT and 5HTTLPR alleles. We suggest that the finding of reduced bi-directionality does not contradict the notion of reduced top-down emotion regulation in low activity carriers as it includes both top-down and bottom-up mechanisms. Thus, our data add a new aspect (that is, feedback mechanisms) to the frontal-limbic relationships.

Therefore, our results are in an agreement with the above studies that were based on correlational methods.

In contrast to the above studies that were testing a coupling between the two a-priori defined regions, we were able to study effective connectivity within the facial emotion-processing circuit that was established empirically. Our findings add to the existing knowledge by showing that the observed reduced connectivity is not just a correlational in nature but involves both top-down and feedback projections.

We emphasize that finding of bi-directional relationship within cortico-limbic network is in agreement with existing experimental literature, ${ }^{56-58}$ supporting 'Iongitudinal' rather than a rigidly hierarchical network models. It has been shown ${ }^{59}$ that cortico-striato-pallido-thalamo-cortical circuitry was arranged as a series of circuits (closed loops).

The recent studies based on Granger connectivity have also demonstrated resting state ${ }^{60,61}$ or task-dependent ${ }^{62}$ uniand bidirectional effective connectivity.

Another important issue that deserves consideration is the emotion-specificity of the genetic effect.

In our study, all emotional expressions, that is, angry, sad, fearful and happy faces activated emotion-processing circuit; however, the genetic effect on connectivity within this circuit was observed in the fearful condition only. As both angry and fearful facial expressions represent threat-related cues, it is important to consider the possible reasons for lack of the genetic effect in angry faces condition.

Although angry faces clearly provide information about the presence of threat, it has been found that the fearful facial expressions (as signaling more ambiguous threat) have been consistently associated with the strongest amygdala activation. This is in accord with the conceptualization of amygdala as a component of vigilance system responding to ambiguous situations of biological relevance. ${ }^{63}$ This was evident in a study combining fMRI with skin conductance recording, ${ }^{64}$ where the fearful (but not angry) faces elicited activation in amygdala-dependent arousal system for fight/flight, which is recognizable fear network. Given a crucial role of serotonin in modulation of the brain processes underlying responses to potential environmental threats, it is conceivable that the processing of fearful faces would be modulated by the serotonin neurotransmission. Indeed, the processing of fearful but not angry faces has been consistently associated with the serotonin metabolism (see review) ${ }^{65}$ In support of our results, the recognition of fearful but not angry faces was modulated by the 5-HTTLPR $S$ vs $L .{ }^{66}$ There is little evidence of a differential effect of dopaminergic transmission on angry vs fearful faces processing. The investigators report the COMT effect on fear processing in general, for example, the COMT met homozygosity was associated with the lack of ability to extinguish conditioned fear, whereas 5-HTTLPR S homozygosity underlied a potentiation of startle reactions. ${ }^{67}$ 
The authors concluded that that the combination of a 5-HTTLPR S allele and COMT met-homozygosity conferred an enhanced risk for acquiring fear that resisted extinction.

These data support our findings of a gene-gene effect of COMT and 5-HTTLPR genotypes on fear processing.

Limitations. The absence of an effect of low activity 5HTTLPR and COMT polymorphisms on BOLD signal in limbic regions needs consideration. The effect of 5-HTTLPR on BOLD signal in the amygdala has been replicated in most of the studies, ${ }^{2}$ although not all of them had sufficient power-as indicated in a meta-analysis. ${ }^{68}$ The effect of COMT val/met polymorphisms on amygdala activation has not been consistently replicated, for example, it was reported by some $^{4}$ but not other investigators. ${ }^{5}$ The authors highlighted an importance of baseline stimuli that was not controlled for in some previous studies. Thus, an exaggerated BOLD signal in amygdala to emotional vs neutral stimuli could have been accounted for by significantly greater amygdala response to the baseline, that is, fixation cross, perceived as an ambiguous signal by $S$-allele carriers. $^{69,70}$ However, a recent study that directly tested the effect of a fixation cross on BOLD response ${ }^{71}$ did not replicate the findings of greater activation to fixation cross relative to the neutral faces.

Dynamic facial stimuli used in our study represent relatively new type of experimental stimuli. Although they provide for closest possible analogy to the socially occurring events, developing an adequate baseline condition proved to be a challenging task. There is little knowledge regarding the perceptual effect of the baseline condition-moving ovalswhich may come across as emotionally ambiguous signals. This may have resulted in a greater amygdala activity in $S$ - and met-allele carriers such that the net BOLD signal changes to emotional faces vs baseline condition was not significantly exaggerated in these individuals. Thus, this should be tested in further research. Alternatively, the absence of a genetic effect on BOLD response in amygdala should not be regarded as a false negative, but rather genuine, statistically plausible negative result. Indeed, a proportion of studies with negative results is expected even if there is a true relationship between COMT (or 5-HTTLPR) and the BOLD response in amygdala to aversive signals. For instance if the statistical power of neuroimaging genetic studies was as high as $90 \%$, still, 1 out of 10 studies should not detect the effect. Therefore, we suggest that the negative results have to be reported in order to avoid the publication bias, which distorts the real state of neuroimaging research.

We emphasize that the main focus of this study was on effective connectivity within the emotion-processing circuit, the measure of which (Granger causality) is based on temporal precedence and thus is not directly related to magnitude of the BOLD effect.

As mentioned above, due to some missing data, we have used the imputation method. To exclude any false positive results due to the imputation, we re-analyzed the data using the data only pertaining to the 84 individuals with full genotyping information. The interaction remained significant: $\mathrm{F}=6.4 \mathrm{df}=3.79 ; P=0.0006$, adjusted $R^{2}=16 \%$.

\section{Conclusion}

Our results indicate that the interaction of 5-HTTLPR and COMT low activity alleles may be associated with reduced reciprocal connectivity within the emotion-processing circuit that includes frontal, temporal, occipital regions and right amygdala. This epistatic effect may underlie an inefficient emotion regulation in these individuals that increases the risk to MDD.

\section{Conflict of interest}

The authors declare no conflict of interest.

Acknowledgements. The study was supported by the Wellcome Trust grant to MLP. JRS thanks FAPESP-Brazil for financial support in Granger causality methods development.

1. Meyer-Lindenberg A, Weinberger DR. Intermediate phenotypes and genetic mechanisms of psychiatric disorders. Nat Rev Neurosci 2006; 7: 818-827.

2. Scharinger C, Rabl U, Sitte HH, Pezawas L. Imaging genetics of mood disorders. Neuroimage 2010; 53: 810-821.

3. Hariri AR, Mattay VS, Tessitore A, Kolachana B, Fera F, Goldman D et al. Serotonin transporter genetic variation and the response of the human amygdala. Science 2002; 297: 400-403.

4. Smolka MN, Schumann G, Wrase J, Grüsser SM, Flor H, Mann K et al. Catechol-Omethyltransferase val158met genotype affects processing of emotional stimuli in the amygdala and prefrontal cortex. J Neurosci 2005; 25: 836-842.

5. Drabant EM, Hariri AR, Meyer-Lindenberg A, Munoz KE, Mattay VS, Kolachana BS et al. Catechol O-methyltransferase val158met genotype and neural mechanisms related to affective arousal and regulation. Arch Gen Psychiatry 2006; 63: 1396-1406.

6. Williams LM, Gatt JM, Grieve SM, Dobson-Stone C, Paul RH, Gordon E et al. COMT Val108/158Met polymorphism effects on emotional brain function and negativity bias. Neuroimage 2010; 53: 918-925.

7. Papaleo F, Crawley JN, Song J, Lipska BK, Pickel J, Weinberger DR et al. Genetic dissection of the role of catechol-O-methyltransferase in cognition and stress reactivity in mice. J Neurosci 2008; 28: 8709-8723.

8. Lopez-Leon S, Janssens ACJW, Gonzalez-Zuloeta Ladd AM, Del-Favero J, Claes SJ, Oostra BA et al. Meta-analyses of genetic studies on major depressive disorder. $\mathrm{Mol}$ Psychiatry 2007; 13: 772-785.

9. Grigorenko EL. Epistasis and the genetics of complex traits. In: Plomin R, DeFries J, Craig IW, McGuffin P (eds). Behavioral Genetics in the Postgenomic Era. American Psychological Association: Washington, DC, USA, 2003, pp 247-266.

10. Opmeer EM, Kortekaas R, Aleman A. Depression and the role of genes involved in dopamine metabolism and signalling. Prog Neurobiol 2010; 92: 112-133.

11. Mandelli L, Serretti A, Marino E, Pirovano A, Calati R, Colombo C. Interaction between serotonin transporter gene, catechol-O-methyltransferase gene and stressful life events in mood disorders. Int J Neuropsychopharmacol 2007; 10: 437-447.

12. Smolka MN, Buhler M, Schumann G, Klein S, Hu XZ, Moayer M et al. Gene-gene effects on central processing of aversive stimuli. Mol Psychiatry 2007; 12: 307-317.

13. Meyer-Lindenberg A. Neural connectivity as an intermediate phenotype: brain networks under genetic control. Hum Brain Mapp 2009; 30: 1938-1946.

14. Pezawas L, Meyer-Lindenberg A, Drabant EM, Verchinski BA, Munoz KE, Kolachana BS. 5-HTTLPR polymorphism impacts human cingulate-amygdala interactions: a genetic susceptibility mechanism for depression. Nat Neurosci 2005; 8: 828-834.

15. Jacques $P$, Dolcos $F$, Cabeza $R$. Effects of aging on functional connectivity of the amygdala during negative evaluation: A network analysis of fMRI data. Neurobiol Aging 2010; 31: 315-327.

16. Anand $A$, Li Y, Wang $Y$, Wu J, Gao S, Bukhari $L$ et al. Activity and connectivity of brain mood regulating circuit in depression: A functional magnetic resonance study. Biol Psychiatry 2005; 57: 1079-1088.

17. Johnstone $\mathrm{T}$, van Reekum CM, Urry HL, Kalin NH, Davidson RJ. Failure to regulate: counterproductive recruitment of top-down prefrontal-subcortical circuitry in major depression. J Neurosci 2007; 27: 8877-8884.

18. Chen $\mathrm{CH}$, Suckling J, Ooi C, Fu CH, Williams SC, Walsh ND et al. Functional coupling of the amygdala in depressed patients treated with antidepressant medication. Neuropsychopharmacology 2008; 33: 1909-1918.

19. Heller AS, Johnstone T, Shackman AJ, Light SN, Peterson MJ, Kolden GG et al. Reduced capacity to sustain positive emotion in major depression reflects diminished maintenance of fronto-striatal brain activation. Proc Natl Acad Sci USA 2011; 106: 22445-22450. 
20. Phillips ML, Drevets WC, Rauch SL, Lane R. Neurobiology of emotion perception II: Implications for major psychiatric disorders. Biol Psychiatry 2003; 54: 515-528.

21. Heinz A, Braus DF, Smolka MN, Wrase J, Puls I, Hermann D et al. Amygdala-prefrontal coupling depends on a genetic variation of the serotonin transporter. Nat Neurosci 2005; 8 : 20-21.

22. Rasch B, Spalek K, Buholzer S, Luechinger R, Boesiger P, de Quervain DJ et al. Aversive stimuli lead to differential amygdala activation and connectivity patterns depending on catechol-O-methyltransferase Val158Met genotype. Neuroimage 2010; 52: 1712-1719.

23. Krugel LK, Biele G, Mohr PN, Li SC, Heekeren HR. Genetic variation in dopaminergic neuromodulation influences the ability to rapidly and flexibly adapt decisions. Proc Natl Acad Sci USA 2009; 106: 17951-17956.

24. Granger CWJ. Investigating causal relations by econometric models and crossspectral methods. Econometrica 1969; 37: 424-438.

25. Goebel R, Roebroeck A, Kim DS, Formisano E. Investigating directed cortical interactions in time-resolved fMRI data using vector autoregressive modeling and Granger causality mapping. Magn Reson Imaging 2003; 21: 1251-1261.

26. Friston KJ, Buechel C, Fink GR, Morris J, Rolls E, Dolan RJ. Psychophysiological and modulatory interactions in neuroimaging. Neuroimage 1997; 6: 218-229.

27. McIntosh AR. Understanding neural interactions in learning and memory using functional neuroimaging. Ann NY Acad Sci 1998; 8: 556-571.

28. Friston K, Harrison L, Penny W. Dynamic causal modelling. Neuroimage 2003; 19: 1273-1302.

29. Almeida JR, Versace A, Mechelli A, Hassel S, Quevedo K, Kupfer DJ et al. Abnormal amygdala-prefrontal effective connectivity to happy faces differentiates bipolar from major depression. Biol Psychiatry 2009; 66: 451-459.

30. Sato JR, Fujita A, Cardoso EF, Thomaz CE, Brammer MJ, Amaro Jr E. Analyzing the connectivity between regions of interest: an approach based on cluster Granger causality for fMRI data analysis. Neuroimage 2010; 52: 1444-1455

31. LaBar KS, Crupain MJ, Voyvodic JT, McCarthy G. Dynamic perception of facial affect and identity in the human brain. Cereb Cortex 2003; 13: 1023-1033.

32. First MB, Gibbon M, Spitzer RL, Williams JBW. SCID-1. Structured Clinical Interview for DSM-IV-TR Axis 1 Disorders. Biometrics Research: New York, NY, 2001.

33. Puls I, Mohr J, Wrase J, Vollstädt-Klein S, Leménager T, Vollmert C et al. A model comparison of COMT effects on central processing of affective stimuli. Neuroimage 2009; 46: 683-691.

34. Meyer-Lindenberg A, Nichols T, Callicott JH, Ding J, Kolachana B, Buckholtz J et al. Impact of complex genetic variation in COMT on human brain function. Mol Psychiatry 2006; 11: 867-877.

35. Nackley AG, Shabalina SA, Tchivileva IE, Satterfield K, Korchynskyi O, Makarov SS et al. Human catechol-O-methyltransferase haplotypes modulate protein expression by altering mRNA secondary structure. Science (New York, NY) 2006; 314: 1930-1933.

36. Heils A, Teufel A, Petri S, Stober G, Riederer P, Bengel D. Allelic variation of human serotonin transporter gene expression. J Neurochem 1996; 66: 2621-2624.

37. Hu XZ, Lipsky RH, Zhu G, Akhtar LA, Taubman J, Greenberg BD et al. Serotonin transporter promoter gain-of-function genotypes are linked to obsessive-compulsive disorder. Am J Hum Genet 2006; 78: 815-826.

38. Parsey RV, Hastings RS, Oquendo MA, Hu X, Goldman D, Huang YY et al. Effect of a triallelic functional polymorphism of the serotonin-transporter-linked promoter region on expression of serotonin transporter in the human brain. Am J Psychiatry 2006; 163: 48-51.

39. Zhao JH, Lissarrague S, Essioux L, Sham PC. Genecounting: haplotype analysis with missing genotypes. Bioinformatics 2002; 18: 1694-1695.

40. Radua J, Mataix-Cols D, Phillips ML, El-Hage W, Kronhaus DM, Cardoner N et al. A new meta-analytic method for neuroimaging studies that combines reported peak coordinates and statistical parametric maps. Eur Psychiatry 2011; doi: 10.1016/j.eurpsy.2011.04.001 (in press)

41. Brammer MJ, Bullmore ET, Simmons A, Williams SC, Grasby PM, Howard RJ et al. Generic brain activation mapping in functional magnetic resonance imaging: A nonparametric approach. Magn Reson Imaging 1997; 15: 763-770.

42. Hamilton JP, Chen G, Thomason ME, Schwartz ME, Gotlib IH. Investigating neural primacy in major depressive disorder: multivariate Granger causality analysis of resting-state fMRI time-series data. Mol Psychiatry 2011; 16: 763-772.

43. Bassett DS, Bullmore ET. Human brain networks in health and disease. Curr Opin Neurol 2009; 22: 340-347.

44. Haxby JV, Hoffman EA, Gobbini MI. The distributed human neural system for face perception. Trends Cogn Sci 2000; 4: 223-233.

45. Said CP, Moore CD, Engell AD, Todorov A, Haxby JV. Distributed representations of dynamic facial expressions in the superior temporal sulcus. J Vis 2010; 10: 1-12.

46. Surguladze SA, Elkin A, Ecker C, Kalidindi S, Corsico A, Giampietro V et al. Genetic variation in the serotonin transporter modulates neural system-wide response to fearful faces. Genes Brain Behav 2008; 7: 543-551.

47. Kim MJ, Loucks RA, Palmer AL, Brown AC, Solomon KM, Marchante AN et al. The structural and functional connectivity of the amygdala: from normal emotion to pathological anxiety. Behav Brain Res 2011; 223: 403-410.

48. Shin LM, Wright $\mathrm{Cl}$, Cannistraro PA, Wedig MM, McMullin $\mathrm{K}$, Martis $\mathrm{B}$ et al. A functional magnetic resonance imaging study of amygdala and medial prefrontal cortex responses to overtly presented fearful faces in posttraumatic stress disorder. Arch Gen Psychiatry 2005; 62: $273-281$

49. Klumpp $\mathrm{H}$, Angstadt $\mathrm{M}$, Phan $\mathrm{KL}$. Insula reactivity and connectivity to anterior cingulate cortex when processing threat in generalized social anxiety disorder. Biol Psychol 2012; 89: 273-276

50. Ghashghaei HT, Barbas H. Pathways for emotion: interactions of prefrontal and anterior temporal pathways in the amygdala of the rhesus monkey. Neuroscience 2002; 115 1261-1279.

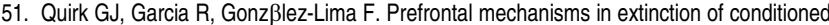
fear. Biol Psychiatry 2006; 60: 337-343.

52. Stein JL, Wiedholz LM, Bassett DS, Weinberger DR, Zink CF, Mattay VS et al. A validated network of effective amygdala connectivity. Neuroimage 2007; 36: 736-745.

53. Rajkowska G, Miguel-Hidalgo JJ. Gliogenesis and glial pathology in depression. CNS Neurol Disord Drug Targets 2007; 6: 219-233.

54. Sheline YI. 3D MRI studies of neuroanatomic changes in unipolar major depression: the role of stress and medical comorbidity. Biol Psychiatry 2000; 48: 791-800.

55. Mayberg HS, Liotti M, Brannan SK, McGinnis S, Mahurin RK, Jerabek PA et al. Reciprocal limbic-cortical function and negative mood: converging PET findings in depression and normal sadness. Am J Psychiatry 1999; 156: 675-682.

56. Aggleton JP, Burton MJ, Passingham RE. Cortical and subcortical afferents to the amygdala of the rhesus monkey (Macaca mulatta). Brain Res 1980; 190: 347-368.

57. Amaral DG, Price JL. Amygdalo-cortical projections in the monkey (Macaca fascicularis). $J$ Comp Neurol 1984; 230: 465-496.

58. Ghashghaei HT, Hilgetag CC, Barbas H. Sequence of information processing for emotions based on the anatomic dialogue between prefrontal cortex and amygdala. Neuroimage 2007; 34: 905-923.

59. Thompson RH, Swanson LW. Hypothesis-driven structural connectivity analysis supports network over hierarchical model of brain architecture. Proc Natl Acad Sci USA 2010; 107: 15235-15239.

60. Liao W, Qiu C, Gentili C, Walter M, Pan Z, Ding J et al. Altered effective connectivity network of the amygdala in social anxiety disorder: a resting-state FMRI study. PLOS ONE 2010; 5: e15238.

61. Deshpande G, Santhanam P, Hu X. Instantaneous and causal connectivity in resting state brain networks derived from functional MRI data. Neuroimage 2011; 54: 1043-1052.

62. Ge T, Feng J, Grabenhorst F, Rolls ET. Componential Granger causality, and its application to identifying the source and mechanisms of the top $\Gamma$ Çôdown biased activation that controls attention to affective vs sensory processing. Neuroimage 2012; 59: 1846-1858.

63. Whalen PJ. Fear, vigilance, and ambiguity: initial neuroimaging studies of the human amygdala. Curr Dir Psychol Sci 1998; 7: 177-187.

64. Williams LM, Das P, Liddell B, Olivieri G, Peduto A, Brammer MJ et al. BOLD, sweat and fears: $\mathrm{AMRI}$ and skin conductance distinguish facial fear signals. Neuroreport 2005; 16 : 49-52.

65. Del-Ben CM, Ferreira CA, Alves-Neto WC, Graeff FG. Serotonergic modulation offaceemotion recognition. Braz J Med Biol Res 2008; 41: 263-269.

66. Szekely E, Herba CM, Arp PP, Uitterlinden AG, Jaddoe VW, Hofman A et al. Recognition of scared faces and the serotonin transporter gene in young children: the Generation R Study J Child Psychol Psychiatry 2011; 52: 1279-1286.

67. Lonsdorf TB, Weike Al, Nikamo P, Schalling M, Hamm AO, Ohman A. Genetic gating of human fear learning and extinction: possible implications for gene-environment interaction in anxiety disorder. Psychol Sci 2009; 20: 198-206.

68. Munafo MR, Brown SM, Hariri AR. Serotonin transporter (5-HTTLPR) genotype and amygdala activation: a meta-analysis. Biol Psychiatry 2008; 63: 852-857.

69. Canli T, Omura K, Haas BW, Fallgatter A, Constable RT, Lesch KP. Beyond affect: A role for genetic variation of the serotonin transporter in neural activation during a cognitive attention task. PNAS 2005; 102: 12224-12229.

70. Heinz A, Smolka MN, Braus DF, Wrase J, Beck A, Flor $\mathrm{H}$ et al. Serotonin transporter genotype (5-HTTLPR): effects of neutral and undefined conditions on amygdala activation. Biol Psychiatry 2007; 61: 1011-1014.

71. von dem Hagen EA, Passamonti L, Nutland S, Sambrook J, Calder AJ. The serotonin transporter gene polymorphism and the effect of baseline on amygdala response to emotional faces. Neuropsychologia 2011; 49: 674-680.

Translational Psychiatry is an open-access journal published by Nature Publishing Group. This work is licensed under the Creative Commons Attribution-Noncommercial-No Derivative Works 3.0 Unported License. To view a copy of this license, visit http://creativecommons.org/licenses/by-nc-nd/3.0/

\section{Supplementary Information accompanies the paper on the Translational Psychiatry website (http://www.nature.com/tp)}

